\title{
Preface to the special issue on learning analytics and personalised support across spaces
}

\author{
Roberto Martinez-Maldonado ${ }^{1}$ - Davinia Hernández-Leo ${ }^{2} \cdot$ Abelardo Pardo $^{3}$
}

Published online: 21 August 2019

(c) Springer Nature B.V. 2019

\section{What is learning across spaces?}

Although the use of educational technology to support online and distance learning is, arguably, a mature field (Broadbent and Poon 2015; Stephenson 2018), students' learning ultimately happens where the student is (Lave and Wenger 1991). Learning rarely happens in a single physical space or using a single digital environment (Looi et al. 2015; Sharples and Roschelle 2010). Recent literature reviews have shown that most of the work in the areas of learning analytics and personalised technologyenhanced learning has focused on modelling student knowledge or skills from interaction traces automatically captured as students use a specific educational system (Abyaa et al. 2019; FitzGerald et al. 2018; Normadhi et al. 2019). Yet, students commonly work outside the boundaries of institutional learning systems. For example, they may interact face-to-face out of school, use educational tools and resources available in the internet, or even use tools that were not originally conceived for educational purposes to complete learning tasks. At the same time, teachers often use a range of tools that reflect the characteristics of their learning designs, which may not necessarily be part of the available set of institutional tools (Prieto et al. 2012). Educational institutions such as schools and universities are frequently deploying and testing a variety of educational technologies and pedagogical resources in both online and face-to-face settings at an institutional level (Rogers 2008). Moreover, learning opportunities often happen in places and at moments that go beyond formal education (Carr et al. 2018).

Roberto Martinez-Maldonado

Roberto.MartinezMaldonado@monash.edu

Davinia Hernández-Leo

Davinia.Hernandez-Leo@upf.edu

Abelardo Pardo

Abelardo.Pardo@unisa.edu.au

1 Monash University, Melbourne, Australia

2 Universitat Pompeu Fabra, Barcelona, Spain

3 University of South Australia, Adelaide, Australia 
From a pedagogical perspective, this combination of tools, resources, and settings presents the potential of new more effective learning experiences. Educational research has revealed the pedagogical benefits of letting students experience different types of content, "real-world" challenges, and physical and social interactions with educators or other students (Delgado-Kloos et al. 2012; Muñoz-Cristóbal et al. 2017; Pérez-Sanagustín et al. 2012). Pervasive and mobile technologies can be used to allow students to access educational resources from different physical spaces (e.g. ubiquitous/mobile learning support) or to enrich their learning experiences in the classroom in ways that were not previously possible (e.g. face-to-face/blended learning support). In parallel, these technologies are increasingly embedded into everyday objects that can communicate information and generate large amounts of interaction data. This tendency creates new possibilities for richer student models that capture the complexity of the learning journeys across physical and digital spaces (Delgado-Kloos et al. 2012). These richer models may, in turn, be used to effectively provide continued, personalised support and automated feedback, moving beyond the idea of providing support only while students interact with a system (Kitto et al. 2015).

In short, learning is happening in an increasing number of spaces, at different times and with the support of different tools and technologies. However, creating mechanisms to model and provide support to learning happening "across spaces" is challenging and still remains underexplored (FitzGerald et al. 2018; MartinezMaldonado et al. 2016). Providing continued support in fully digital spaces (e.g. for mobile and web-based experiences) has been approached to different extents (PérezSanagustín et al. 2012; Prieto et al. 2012). An emerging challenge is how to create analytics innovations that can provide adapted or personalised support for students' learning considering that learning happens in different types of spaces, both physical and digital.

\section{Definitions}

This special issue responds to the growing interest in this theme as observed in a series of workshops, titled Learning Analytics Across Spaces (CrossLAK), organised at the International Conference on Learning Analytics and Knowledge in 2016 and 2017 (Martinez-Maldonado et al. 2016). This workshop later merged with the Multimodal Learning Analytics (MMLA) series of workshops and was re-launched as the CrossMMLA initiative. The interest shown by researchers and practitioners has led to the establishment of the SoLAR Special Interest Group on Multimodal Learning Analytics Across Spaces. However, CrossLAK and MMLA perspectives follow different philosophical and practical approaches.

Learning Analytics and Personalised Support Across Spaces (CrossLAK) follows a top-down approach, focusing on educational questions or problems first and then on the evidence that needs to be captured, modelled, or analysed to address those (Delgado-Kloos et al. 2012; Martinez-Maldonado et al. 2016). It embraces the complexity of learning phenomena as human activity that is distributed across spaces, people, tools (both digital and physical), and time. Once the learning problem has 
been identified, a CrossLAK initiative would focus on assessing the feasibility of using learning analytics and modelling to tackle such a problem. These analytics may be simple (for example, just focused on the analysis of one modality of data that is collected from several spaces or that is relevant to achieve personalisation across several spaces) or quite sophisticated (for example, requiring the capture of traces such as eye gaze, posture, positioning, speech, and physiological markers). Since the focus is on learning happening in authentic spaces, the philosophical intention is to apply analytics in-the-wild rather than in-the-laboratory.

By contrast, MMLA favours a bottom-up approach where the focus is on using multiple streams of data, moving beyond the analysis of clickstreams and keystrokes. Thus, MMLA is currently focused on exploring the kinds of insights sensing technologies may provide about learning processes (Blikstein and Worsley 2018; Ochoa 2017). MMLA leverages the increasingly widespread availability of sensors and high-frequency data collection technologies to enrich the existing data available. The main rationale is to obtain more complete models of the learners by sensing other modalities of interaction (e.g. posture, gaze, gesture, and verbal communication). Because of this, experimental or controlled setups are commonly used to test theory (e.g. Schneider and Pea 2017) or to explore potential relationships between sources of data (e.g. Malmberg et al. 2018). Using such technologies, in combination with machine learning and artificial intelligence techniques, a number of solutions can be offered for ubiquitous learning.

Although CrossLAK and MMLA have some elements that distinguish them from each other, they both share the common aim of deploying learning analytics and personalisation innovations that can be used across diverse authentic learning environments whilst students feature various modalities of interaction or behaviour. Both approaches are aimed at taking advantage of emerging sensing and data processing technologies to gain a deeper understanding of learning, build more complete models of students, and move beyond the analysis of clickstreams and keystrokes by also considering other sources of evidence such as speech, handwriting, sketching, gestures, postures, affective states, or eye gazing. Finally, both approaches value the use of educational theory to explain findings and disentangle the intertwined features that can be obtained by analysing multiple streams of heterogeneous data.

In sum, Learning Analytics and Personalised Support Across Spaces involves the consideration of the physical and digital spaces in which students interact and the capture and analysis of sources of evidence that can respond to higher-order, contextualised educational questions or that can serve to provide personalised support effectively.

\section{About this special issue}

This special issue of the Journal of User Modeling and User-Adapted Interaction (UMUAI) was convened to give further identity to the CrossLAK theme, inviting researchers to submit empirical, theoretical, and methodological contributions on topics including but not limited to: 
- Providing personalised support to learners interacting across multiple digital spaces Studies of novel analytics approaches and systems providing personalised support or feedback across multiple digital learning tools. This includes data mining, learner modelling, and/or visualisation (or Open Learner Modelling) approaches applied to datasets that integrate logs from multiple learning tools;

- Providing personalised support bridging the physical and digital realms Design and study of learning situations that include collocated/face-to-face interaction and/or the use of online (remote access) tools (e.g. including "everyday" settings, collocated collaboration situations, multi-device ecologies, ubiquitous learning technology, or blended learning cases); and

- Generating models of learner's interaction data from heterogeneous sources Discussion of methodologies and theoretical approaches and their technical solutions, to acquire learner models by integrating activity logs from multiple sources of student's data. This includes technical approaches (such as conceptual models, formal representation of heterogeneous learner models, or generic user modelling systems) but also non-technical issues (such as privacy and security of information for personalisation, cultural adaptation, or data management).

From 15 expressions of interest, in the form of extended abstracts, seven submissions were received of which four research papers completed peer review, providing an interesting, albeit incomplete, snapshot of Learning Analytics and Personalised Support Across Spaces practical research. Some of these papers address the above topics, while others introduce new ones. Table 1 summarises the papers in this special section.

In the paper titled Detecting Students-at-Risk in Computer Programming Classes with Learning Analytics from Students' Digital Footprints, Azcona et al. (2019) propose a novel methodology to automatically detect students at risk of failing an assignment in computer programming courses and to provide weekly adaptive feedback for both students and lecturers in large classes to react upon. Authors embraced the spirit of learning across spaces by creating models that involve sets of static information about the students, such as demographics and prior academic history, and behavioural logs captured from two different digital spaces: a specialised programming tool and the institutional learning management system (LMS). This paper illustrates the potential of harnessing more sources of evidence about students' behaviour in more than one digital space to build more effective predictive analytics systems that can be used in blended classroom settings.

In the paper titled Exploring Emotional and Cognitive Dynamics of Knowledge Building in Grades 1 and 2, Zhu et al. (2019) studied the co-occurrence of certain emotions and idea generation improvement in the context of knowledge building supported by both physical artefacts and a digital tool. Authors performed a qualitative analysis of learning tasks happening in the classroom and, then, a digital space, to identify how students', according to their level of participation, feature different emotional traits (identified via video coding and sentiment analysis respectively) and how these are associated to the improvement of ideas. This paper illustrates the importance of grounding on well-established theory (e.g. knowledge building) for driving the analysis of logged behaviour and emotional dynamics. Although the analysis of the data was 


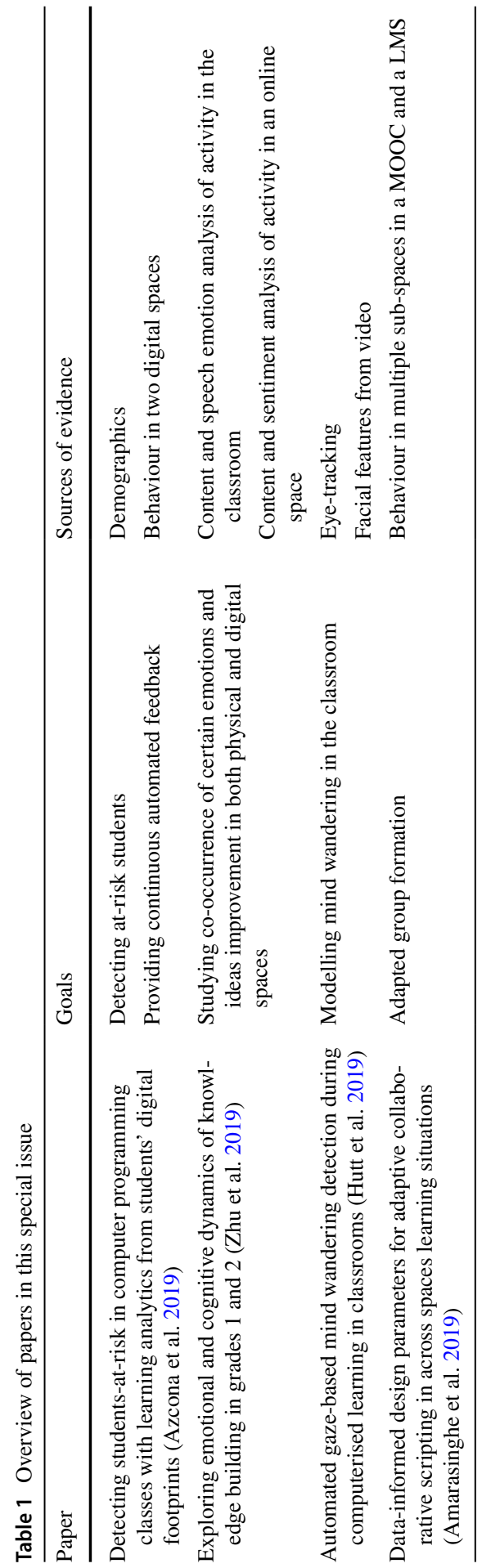


mostly conducted manually, this paper also points at the potential of using emerging multimodal sensors to study multiple dimensions related to knowledge building (e.g. emotional and cognitive) in both physical and digital spaces.

In the paper titled Automated Gaze-Based Mind Wandering Detection during Computerised Learning in Classrooms, Hutt et al. (2019) investigate the potential of using commercial off-the-shelf (COTS) eye-trackers to detect students' mind wandering. Authors present five studies, with students using an intelligent tutoring system, that demonstrate how COTS can be effectively deployed in classroom settings to detect mind wandering and how eye-tracking data can be enriched with features obtained from video recordings to more accurately detect mind wandering periods. This paper illustrates how emerging logging technologies, once thought to be exclusively used in experimental settings, are being successfully deployed in authentic physical classroom spaces. The main contribution of this paper is to set the basis towards developing gazebased attention-aware learning technologies to increase engagement and learning by addressing mind wandering in the classroom.

In the paper titled Data-Informed Design Parameters for Adaptive Collaborative Scripting in Across Spaces Learning Situations, Amarasinghe et al. (2019) demonstrate how traces of students' behaviour using multiple educational tools can be used to predict future participation of students working in groups. Authors apply predictive analytics on data collected from different learning spaces, associated with both individual and collaborative learning activity engagement, to propose an automated group formation strategy. Two case studies are presented in the contexts of a MOOC (Massive Open Online Course) and the institutional LMS. This paper illustrates how predictive analytics can be used to analyse students' behavioural activity across spaces to inform the formulation of adaptive learning groups in real time.

\section{Conclusions and future avenues of research}

The four papers in this special issue showcase studies conducted in authentic blended learning settings. The educational questions addressed in these papers illustrate that an analytical approach across spaces can serve to address well-known educational problems (e.g. detecting at-risk students and group formation) or aspects of learning that have not been deeply studied (e.g. modelling mind wandering and co-occurrence of emotion and ideation). The studies also showcase that an across spaces stance may involve the analysis of behavioural data captured through online systems but also the use of emerging (multimodal) sensors. Clearly, this research area is still in its maturing stage. The rapid changes in sensor technology and its cost offer tremendous opportunities to harness their potential and translate this potential into meaningful improvements to support learning where it actually occurs.

\section{References}

Abyaa, A., Idrissi, M.K., Bennani, S.: Learner modelling: systematic review of the literature from the last 5 years. Educ. Technol. Res. Dev. (2019). https://doi.org/10.1007/s11423-018-09644-1 
Amarasinghe, I., Hernández-Leo, D., Jonsson, A.: Data-informed design parameters for adaptive collaborative scripting in across-spaces learning situations. User Model. User Adapt. Interact. (2019). https ://doi.org/10.1007/s11257-019-09233-8

Azcona, D., Hsiao, I.-H., Smeaton, A.F.: Detecting students-at-risk in computer programming classes with learning analytics from students' digital footprints. User Model. User Adapt. Interact. (2019). https://doi.org/10.1007/s11257-019-09234-7

Blikstein, P., Worsley, M.: Multimodal learning analytics and assessment of open-ended artifacts. In: Niemi, D., Pea, R.D., Saxberg, B., Clark, R.E. (eds.) Learning Analytics in Education, p. 89. IAP, Charlotte (2018)

Broadbent, J., Poon, W.: Self-regulated learning strategies and academic achievement in online higher education learning environments: a systematic review. Internet High. Educ. 27, 1-13 (2015)

Carr, A., Balasubramanian, K., Atieno, R., Onyango, J.: Lifelong learning to empowerment: beyond formal education. Distance Educ. 39(1), 69-86 (2018)

Delgado-Kloos, C., Hernández-Leo, D., Asensio-Pérez, J.I.: Technology for learning across physical and virtual spaces J UCS special issue. J. Univers. Comput. Sci. 18(15), 2093-2096 (2012)

FitzGerald, E., Jones, A., Kucirkova, N., Scanlon, E.: A literature synthesis of personalised technologyenhanced learning: what works and why. Res. Learn. Technol. (2018). https://doi.org/10.25304/rlt. v26.2095

Hutt, S., Krasich, K., Mills, C., Bosch, N., White, S.M., Brockmole, J.R., D’Mello, S.K.: Automated gaze-based mind wandering detection during computerized learning in classrooms. User Model. User Adapt. Interact. (2019). https://doi.org/10.1007/s11257-019-09228-5

Kitto, K., Cross, S., Waters, Z., Lupton, M.: Learning analytics beyond the LMS: the connected learning analytics toolkit. In: Proceedings of the Fifth International Conference on Learning Analytics and Knowledge, pp. 11-15. ACM, Poughkeepsie (2015)

Lave, J., Wenger, E.: Situated Learning: Legitimate Peripheral Participation. Cambridge University Press, Cambridge (1991)

Looi, C.-K., Wong, L.-H., Milrad, M.: Guest editorial: special issue on seamless, ubiquitous, and contextual learning. IEEE Trans. Learn. Technol. 8(1), 2-4 (2015)

Malmberg, J., Järvelä, S., Holappa, J., Haataja, E., Huang, X., Siipo, A.: Going beyond what is visible: What multichannel data can reveal about interaction in the context of collaborative learning? Comput. Hum Behav. 96, 235-245 (2018)

Martinez-Maldonado, R., Hernandez-Leo, D., Pardo, A., Suthers, D., Kitto, K., Charleer, S., Aljohani, N.R., Ogata, H.: Cross-LAK: learning analytics across physical and digital spaces. In: Paper Presented at the Proceedings of the Sixth International Conference on Learning Analytics and Knowledge, pp. 486-487. ACM (2016)

Muñoz-Cristóbal, J.A., Rodriguez Triana, M., Bote-Lorenzo, M.L., Villagrá-Sobrino, S.L., AsensioPérez, J.I., Martínez-Monés, A.: Toward multimodal analytics in ubiquitous learning environments. In: Paper Presented at the Joint Proceedings of the Sixth Multimodal Learning Analytics (MMLA) Workshop and the Second Cross-LAK Workshop co-located with 7th International Learning Analytics and Knowledge Conference, pp. 60-67. CEUR (2017)

Normadhi, N.B.A., Shuib, L., Nasir, H.N.M., Bimba, A., Idris, N., Balakrishnan, V.: Identification of personal traits in adaptive learning environment: systematic literature review. Comput. Educ. 130, 168-190 (2019)

Ochoa, X.: Multimodal learning analytics. In: Lang, C., Siemens, G., Wise, A.F., Gaševic, D. (eds.) The Handbook of Learning Analytics, pp. 129-141. Solar, Alberta (2017)

Pérez-Sanagustín, M., Santos, P., Hernández-Leo, D., Blat, J.: 4SPPIces: a case study of factors in a scripted collaborative-learning blended course across spatial locations. Int. J. Comput. Support. Collab. Learn. 7(3), 443-465 (2012)

Prieto, L.P., Muñoz-Cristóbal, J.A., Asensio-Pérez, J.I., Dimitriadis, Y.: Making learning designs happen in distributed learning environments with GLUE!-PS. In: Ravenscroft, A., Lindstaedt, S., Kloos, C., Hernández-Leo, D. (eds.) 21st Century Learning for 21st Century Skills, pp. 489-494. Springer, Berlin (2012)

Rogers, Y.: Using external visualizations to extend and integrate learning in mobile and classroom settings. In: Gilbert, J., Reiner, M., Nakhleh, M. (eds.) Visualization: Theory and Practice in Science Education, pp. 89-102. Springer, Dordrecht (2008)

Schneider, B., Pea, R.: Real-time mutual gaze perception enhances collaborative learning and collaboration quality. Educ. Med. Technol. Yearb. 40, 99-125 (2017) 
Sharples, M., Roschelle, J.: Guest editorial: special section on mobile and ubiquitous technologies for learning. IEEE Trans. Learn. Technol. 1, 4-6 (2010)

Stephenson, J.: Teaching and Learning Online: New Pedagogies for New Technologies. Routledge, Abingdon (2018)

Zhu, G., Xing, W., Costa, S., Scardamalia, M., \& Pei, B.: Exploring emotional and cognitive dynamics of knowledge building in grades 1 and 2. User Model. User Adapt. Interact. (2019). https://doi. org/10.1007/s11257-019-09241-8

Publisher's Note Springer Nature remains neutral with regard to jurisdictional claims in published maps and institutional affiliations.

Roberto Martinez-Maldonado is a Senior Lecturer at Monash University, in Melbourne, Australia. He has a background in Computing Engineering. His areas of research include human-computer interaction, learning analytics, artificial intelligence in education, and collaborative learning (CSCL). In the past years, his research has focused on applying artificial intelligence and visualisation techniques to help understand how people learn and collaborate in collocated environments; empowering people with emerging technologies such as interactive surfaces; combining available technologies for capturing traces of collaboration; and helping teachers to orchestrate their classroom through the use of interactive devices and learning analytics innovations.

Davinia Hernández-Leo is Associate Professor and Serra Húnter Fellow at the Department of Information and Communications Technologies Department (DTIC) at UPF, the coordinator of the Interactive and Distributed Technologies for Education group (TIDE), Vice-Dean of the UPF Engineering School, and the Head of its Unit for Teaching Quality and Innovation. Her research lies at the intersection of humancomputer interaction, network and computer applications, and learning sciences, with emphasis on learning design technologies, CSCL, community platforms, data analytics, and architectures and devices for learning. Currently, she is the Vice-President of the European Association for Technology-Enhanced Learning, a member of the editorial board of the IEEE Transactions of Learning Technologies and the Steering Committee of the European Conference on Technology-Enhanced Learning.

Abelardo Pardo is Professor and Dean Academic at the Division of Information Technology, Engineering and the Environment at the University of South Australia. His research interests include the design and deployment of technology to increase the understanding and improve digital learning experiences. More specifically, his work examines the areas of learning analytics, personalised active learning, and technology for student support. 